\title{
Relationship of plasma extracellular-superoxide dismutase level with insulin resistance in type 2 diabetic patients
}

\author{
T Adachi, M Inoue ${ }^{1}$, H Hara, E Maehata ${ }^{2}$ and S Suzuki ${ }^{1}$ \\ Laboratory of Clinical Pharmaceutics, Gifu Pharmaceutical University, Gifu 502-8585, Japan \\ ${ }^{1}$ Division of Endocrinology and Metabolism, Department of Internal Medicine, Showa University Fujigaoka Hospital, Yokohama, Kanagawa 227-8501, Japan \\ ${ }^{2}$ Central Clinical Laboratory, Mitsui Memorial Hospital, Tokyo 101-8643, Japan \\ (Requests for offprints should be addressed to T Adachi; Email: adachi@gifu-pu.ac.jp)
}

\begin{abstract}
Extracellular-superoxide dismutase (EC-SOD) is a secretory glycoprotein located in blood vessel walls at high levels and may be important in the antioxidant capability of vascular walls. The aim of this study was to assess plasma levels of EC-SOD and to evaluate the relationship of the EC-SOD level with insulin resistance in type 2 diabetic patients. We determined plasma EC-SOD in 122 patients and found for the first time that the EC-SOD level was strongly and positively related to adiponectin $(r=0.503$, $P<0 \cdot 001$ ), and significantly and inversely related to fasting plasma glucose (FPG) $(r=-0 \cdot 209, P=0 \cdot 022)$, body-mass index (BMI) $(r=-0 \cdot 187, \quad P=0 \cdot 040)$ and homeostasis model assessment-insulin resistance index (HOMA-R) $(r=-0 \cdot 190, \quad P=0 \cdot 039)$. Stepwise-multiple regression
\end{abstract}

analysis also showed a significant influence of adiponectin $(F=33 \cdot 27)$ on the EC-SOD level. Administration of pioglitazone to 19 diabetic patients significantly increased the plasma levels of EC-SOD $(69 \cdot 9 \pm 19 \cdot 3 \mathrm{ng} / \mathrm{ml}$ to $97 \cdot 4 \pm 25.9 \mathrm{ng} / \mathrm{ml} ; P<0 \cdot 0001)$ and adiponectin, while it decreased tumor necrosis factor- $\alpha$ (TNF- $\alpha$ ). The present observations suggest that factors related to the pathogenesis of insulin resistance play an important role in the regulation of the plasma EC-SOD concentration. It is possible that the increase in the EC-SOD level by pioglitazone administration in diabetic patients is due to a decline of TNF- $\alpha$, which is known to suppress EC-SOD expression. Journal of Endocrinology (2004) 181, 413-417

\section{Introduction}

Insulin resistance, which is characterized by a reduced sensitivity of target tissues to insulin, is an important risk factor associated with atherosclerosis (Matsuzawa et al. 1999). Abnormalities in lipid and glucose metabolism are thought to contribute to atherosclerotic vascular damage in the pathologic condition of insulin resistance (Pieper et al. 1995). Diabetic hyperglycemia has been reported to promote the overproduction of reactive oxygen species such as superoxide, causing hyperglycemic vascular damage in vivo (Faure et al. 1999) and in vitro (Nishikawa et al. 2000). Superoxide is secreted from various cells in the vascular system (Ohara et al. 1993) and is implicated in the insulin-resistant state (Bertelsen et al. 2001). The increase in superoxide may contribute to oxidative processes in the vessel wall, such as induction and enhancement of cell membrane lipid peroxidation (Jain 1989) and oxidation of low-density lipoprotein (LDL) (Heinecke et al. 1986). Moreover, superoxide reacts extremely rapidly with nitric oxide (NO), which modulates vasomotor tone, inhibits platelet- and leukocyte-aggregation, and produces peroxynitrite (Koppenol et al. 1992). An increase in superoxide dismutase (SOD) has been reported to attenuate diabetic vascular dysfunction (Craven et al. 2001).

The vascular wall contains large amounts of extracellular-superoxide dismutase (EC-SOD), one of the SOD isozymes produced and secreted to the extracellular space by fibroblasts and smooth muscle cells (Marklund 1990). EC-SOD has an affinity for heparin-like substances (Adachi \& Marklund 1989) and glycosaminoglycans on the endothelial cell surface (Adachi et al. 1996). After secretion, the EC-SOD probably diffuses slowly in the vascular wall and distributes itself according to the amount and affinity of the heparin-like glycosaminoglycans present in the vessel wall (Strålin et al. 1995). The presence of EC-SOD on the endothelial cell surface at a high level might have an important protective effect against superoxide in the vascular wall, supporting the antioxidant role of NO (Wink et al. 1993), and a role in prevention of LDL oxidation (Takatsu et al. 2001). EC-SOD expression in cultured cell lines is known to be regulated by numerous substances such as cytokines. For example, tumor necrosis factor (TNF) $\alpha$ is one of the suppressive factors of EC-SOD expression in vascular cells (Marklund 1992, Strålin \& Marklund 2000). On the other hand, TNF- $\alpha$ is 
Table 1 Clinical characteristics of the study population

(A) Plasma concentration

\begin{tabular}{|c|c|}
\hline Male & Female \\
\hline $81 \cdot 9 \pm 20 \cdot 8$ & $104 \cdot 4 \pm 33 \cdot 2^{* *}$ \\
\hline $8 \cdot 2 \pm 2 \cdot 0$ & $8 \cdot 0 \pm 2 \cdot 0$ \\
\hline $7 \cdot 0 \pm 1 \cdot 1$ & $7 \cdot 1 \pm 1 \cdot 3$ \\
\hline $8 \cdot 4 \pm 6 \cdot 6$ & $10 \cdot 2 \pm 7 \cdot 4$ \\
\hline $23 \cdot 3 \pm 2 \cdot 2$ & $24 \cdot 7 \pm 4 \cdot 6$ \\
\hline $3 \cdot 1 \pm 3 \cdot 1$ & $3 \cdot 6 \pm 2 \cdot 7$ \\
\hline $6 \cdot 5 \pm 3 \cdot 2$ & $9 \cdot 1 \pm 5 \cdot 1^{* *}$ \\
\hline $196 \cdot 7 \pm 30 \cdot 7$ & $210 \cdot 7 \pm 36 \cdot 3^{*}$ \\
\hline $51 \cdot 8 \pm 10 \cdot 5$ & $54 \cdot 9 \pm 12 \cdot 6$ \\
\hline $124 \cdot 2 \pm 27 \cdot 6$ & $131 \cdot 6 \pm 34 \cdot 0$ \\
\hline $111 \cdot 7 \pm 54 \cdot 4$ & $123 \cdot 3 \pm 66 \cdot 6$ \\
\hline
\end{tabular}

(B)

Relation vs EC-SOD

\begin{tabular}{|c|c|c|c|c|c|}
\hline \multicolumn{2}{|l|}{ Male } & \multicolumn{2}{|l|}{ Female } & \multicolumn{2}{|l|}{ Total } \\
\hline$r$ & $F$ & $r$ & $F$ & $r$ & $F$ \\
\hline-0.072 & - & $-0.390 \dagger$ & $6 \cdot 41$ & $-0 \cdot 209 \dagger$ & $4 \cdot 59$ \\
\hline-0.073 & - & $-0 \cdot 210$ & - & -0.099 & - \\
\hline 0.019 & - & $-0.445+\dagger$ & $7 \cdot 63$ & $-0 \cdot 133$ & - \\
\hline$-0 \cdot 174$ & - & $-0.370 \dagger$ & $5 \cdot 73$ & $-0 \cdot 187 \dagger$ & - \\
\hline-0.072 & - & $-0.506+\dagger$ & $10 \cdot 95$ & $-0 \cdot 190 \dagger$ & - \\
\hline 0.380†† & $13 \cdot 3$ & 0.490†† & $9 \cdot 55$ & 0.503†十 & $33 \cdot 27$ \\
\hline$-0 \cdot 117$ & - & -0.163 & - & -0.048 & - \\
\hline-0.020 & - & $0 \cdot 152$ & - & $-0 \cdot 107$ & - \\
\hline-0.135 & - & $-0 \cdot 250$ & - & $-0 \cdot 129$ & - \\
\hline $0 \cdot 118$ & - & $0 \cdot 148$ & - & $0 \cdot 026$ & - \\
\hline
\end{tabular}

(A) Data are presented as mean \pm S.D. Significant differences between males and females $\left({ }^{*} P<0 \cdot 05, * * P<0 \cdot 01\right)$ were analyzed with Student's $t$-test or Welch's $t$-test. (B) Data are correlation coefficients $(r)$ between EC-SOD and other characteristics, and significant relationships are shown with symbols $(\dagger P<0 \cdot 05$, $\dagger+P<0 \cdot 01)$. Stepwise-multiple regression analysis was performed, and significant relationships $(F>4 \cdot 0)$ are shown.

a proposed mediator of insulin resistance, overexpressed in adipose tissue and skeletal muscle of obese and type 2 diabetic subjects (Iwata et al. 2001).

The present study was undertaken to clarify the relationship between the plasma EC-SOD level and indices of insulin-resistance, and the change in the EC-SOD level in insulin-resistant patients by administration of pioglitazone, a thiazolidinedione (TZD) insulin-sensitizing drug.

\section{Materials and methods}

\section{Study population}

One hundred and twenty-two diabetic patients (82 men, average age $59 \cdot 9 \pm 8.8$ years, and 40 women, average age $58 \cdot 4 \pm 12 \cdot 2$ years) who showed a normal plasma creatinine level of below $1.1 \mathrm{mg} / \mathrm{dl}$ and did not receive insulin or pioglitazone treatment were eligible for this study on the relationship between the plasma EC-SOD level and characteristics of insulin resistance. The study on the change in the plasma EC-SOD level by pioglitazone treatment included 19 diabetic patients $(10$ men and nine women). They showed higher diabetes/insulin resistanceparameters: for example, fasting plasma glucose (FPG) of $8 \cdot 88 \pm 2.79 \mathrm{mM}$; hemoglobin $\mathrm{A}_{1 \mathrm{c}}\left(\mathrm{HbA}_{1 \mathrm{c}}\right)$ of $8.5 \pm 1 \cdot 2 \%$ and homeostasis model assessment-insulin resistance index (HOMA-R) of $6 \cdot 7 \pm 8 \cdot 7$. The subjects were administered $15 \mathrm{mg}$ (female patients) or $30 \mathrm{mg}$ (male patients) of pioglitazone once daily. All the study subjects provided written informed consent according to the institutional guidelines.

\section{Laboratory measurements}

Fasting blood samples were obtained. The EC-SOD concentrations in the plasma were determined by ELISA described in our previous reports (Adachi et al. 1994) with minor modifications. The plasma adiponectin concentration was assayed with an ELISA kit (Otsuka Pharmaceutical, Tokyo, Japan). The plasma TNF- $\alpha$ concentration was assayed with a Quantikine HS immunoassay kit ( R \& D Systems, Minneapolis, MN, USA). Other characteristics were measured by standard clinical laboratory methods.

\section{Statistical analysis}

Data were analyzed with the $t$-test (Student's $t$-test or Welch's t-test) and the Wilcoxon signed-rank test. A stepwise multiple-regression analysis was performed to assess the influence of variables on EC-SOD concentrations. Variables were deleted from the regression analysis if the $F$ value was not significant $(<4 \cdot 0)$ at any step of the calculations. A $P$ value less than 0.05 was considered significant.

\section{Results}

\section{Plasma EC-SOD level in diabetic patients}

We assayed the plasma EC-SOD level in 122 diabetic patients (average \pm S.D. of EC-SOD level: $89.5 \pm 27.5 \mathrm{ng}$ / $\mathrm{ml})$ and found the level was higher $(P<0 \cdot 001)$ in females $(104 \cdot 4 \pm 33 \cdot 2 \mathrm{ng} / \mathrm{ml})$ than in males $(81 \cdot 9 \pm 20 \cdot 8 \mathrm{ng} / \mathrm{ml})$. The plasma adiponectin level was lower in males than in females, consistent with a previous report (Nishizawa et al. 2002), whereas there were no significant gender differences in FPG, $\mathrm{HbA}_{1 \mathrm{c}}$, immunoreactive insulin (IRI), body-mass index (BMI) and HOMA-R, as shown in Table 1. The plasma EC-SOD levels were significantly and inversely related to FPG $(r=-0.209, P=0.022)$, 


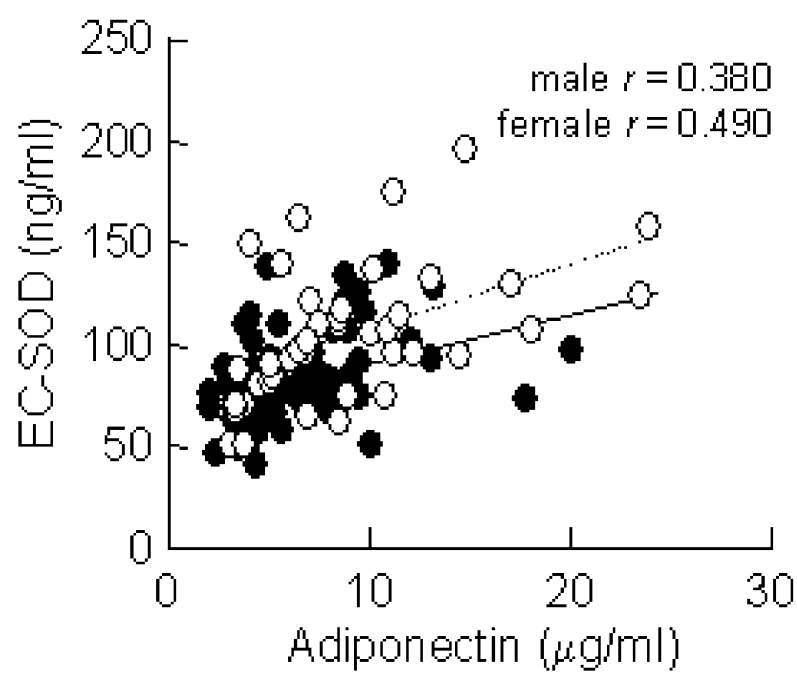

Figure 1 Relationship between the plasma EC-SOD and adiponectin levels. White circles and black circles show female and male patients respectively. The regression lines are presented as a solid line for the male group and a dotted line for the female group.

BMI $(r=-0 \cdot 187, P=0 \cdot 040)$ and HOMA-R $(r=-0 \cdot 190$, $P=0 \cdot 039)$, whereas they were strongly and positively related to adiponectin $(r=0.503, \quad P<0.001)$. Stepwise multiple-regression analysis showed a significant relationship between EC-SOD and adiponectin $(F=33 \cdot 27)$, and FPG $(F=4 \cdot 59)$. There were no significant correlations between EC-SOD and $\mathrm{HbA}_{1 c}(r=-0.099)$ or IRI $(r=-0 \cdot 133)$. In men, there was a significant positive correlation between EC-SOD and adiponectin $(r=0 \cdot 380$, $P<0 \cdot 001, F=13 \cdot 03$ ) (Fig. 1). On the other hand, ECSOD in women showed a significant positive correlation with adiponectin $(r=0.490, P=0.001, F=9 \cdot 55)$ (Fig. 1) and negative correlations with FPG $(r=-0 \cdot 390$, $P=0 \cdot 013, F=6 \cdot 41)$, IRI $(r=-0 \cdot 445, P=0 \cdot 005, F=7 \cdot 63)$, BMI $(r=-0.370, P=0.019, F=5.73)$ and HOMA-R $(r=-0.506, P=0 \cdot 001, F=10 \cdot 95)$. There were no significant correlations between EC-SOD and lipid parameters such as total cholesterol (TC), HDL-cholesterol (HDL-C), LDL-cholesterol (LDL-C) and triglyceride (TG).

\section{Change in the plasma EC-SOD level by pioglitazone treatment}

We investigated the change in the plasma EC-SOD level by treatment with the TZD antidiabetic agent pioglitazone. As shown in Fig. 2A, daily administration of pioglitazone to 19 patients for about 3 months significantly increased their plasma EC-SOD concentration $(69.9 \pm 19.3 \mathrm{ng} / \mathrm{ml}$ to $97.4 \pm 25.9 \mathrm{ng} / \mathrm{ml}, \quad P<0.0001)$. Furthermore, we observed a significant increase in the plasma adiponectin concentration $(5 \cdot 1 \pm 1.9 \mu \mathrm{g} / \mathrm{ml}$ to $15 \cdot 8 \pm 7 \cdot 8 \mu \mathrm{g} / \mathrm{ml}, P<0 \cdot 0001)$ (Fig. $2 \mathrm{~B}$ ), as consistent with a previous report (Iwata et al. 2001). Moreover, the plasma TNF- $\alpha$ level was significantly decreased by the pioglitazone treatment $(2.4 \pm 0.7 \mu \mathrm{g} / \mathrm{ml}$ to $1.9 \pm 0.4 \mu \mathrm{g} / \mathrm{ml}$; $P<0 \cdot 0001)$, as shown in Fig. 2C. On the other hand, the subjects $(n=7)$ in the nonpioglitazone-treatment group did not show any significant changes in their plasma EC-SOD, adiponectin and TNF- $\alpha$ levels (Fig. 2A-C).

\section{Discussion}

The present study demonstrates for the first time that plasma EC-SOD in patients with type 2 diabetes were significantly positively correlated with adiponectin and inversely related to indices of insulin resistance.
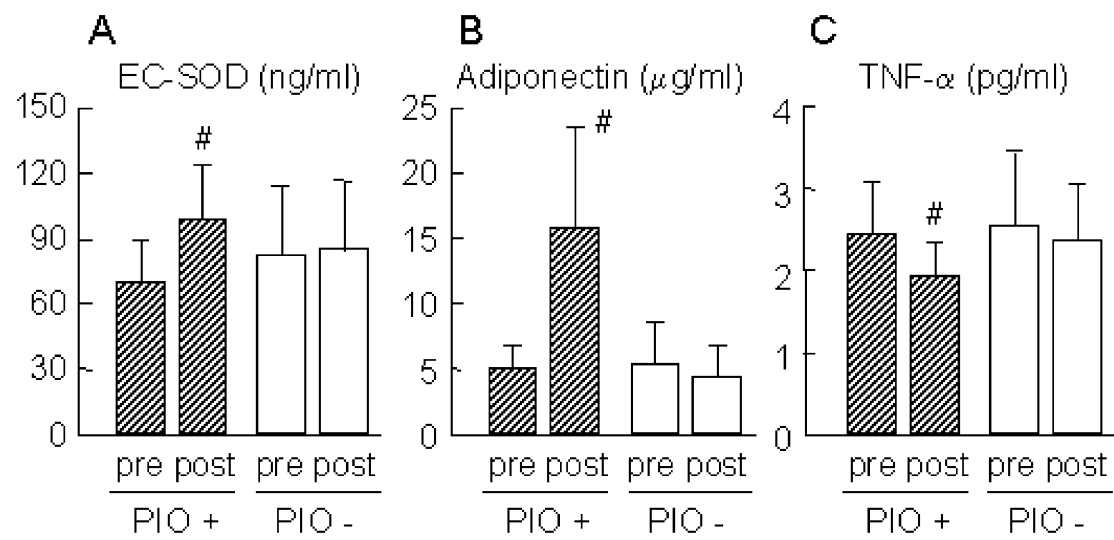

Figure 2 Comparison of the plasma EC-SOD (A), adiponectin (B) and TNF- $\alpha$ (C) levels before and after treatment with pioglitazone. The data are presented as mean \pm S.D. for the pioglitazone-treated group ( $\mathrm{PIO}+, n=19)$ and pioglitazone-untreated group ( $\mathrm{PIO}-, n=7$ ). Significant differences $(P<0 \cdot 0001)$ compared with before treatment were analyzed by the Wilcoxon signed-rank test. 
EC-SOD is the major SOD isozyme in extracellular fluids but is distributed mainly in the blood vessel wall (Strålin et al. 1995). In the vasculature, EC-SOD forms an equilibrium between the plasma phase and endothelial cell surface (Karlsson \& Marklund 1988), and the change in the plasma EC-SOD level might reflect the changes in the expression of EC-SOD and/or EC-SOD-tissue binding. In this study, parameters associated with diabetes/insulin resistance showed more significant relationships to ECSOD in female subjects than in male subjects (Table 1). It has been reported that cardiovascular risk factors, including male sex, high BMI and smoking, decreased the plasma EC-SOD level in an unselected middle-aged population (Marklund et al. 1997). The plasma levels of EC-SOD may be modulated by lifestyle and show a complex covariation with many of the conventional cardiovascular risk factors. From these observations, it is speculated that the cardiovascular risk factors obscure the relationship between EC-SOD and parameters associated with insulin resistance, especially in male subjects.

The major observation indicated in Table 1 is that plasma EC-SOD levels in diabetic patients were strongly positively correlated with adiponectin, whereas they were significantly inversely related to parameters associated with insulin resistance. Moreover, stepwise multiple-regression analyses showed that adiponectin especially highly affects EC-SOD level. The plasma level of adiponectin has been reported to relate inversely to BMI and HOMA-R (Hotta et al. 2000). The plasma EC-SOD level was also inversely related to the above parameters, suggesting that factors related to the pathogenesis of insulin resistance play an important role in the regulation of the plasma EC-SOD. Increases in the plasma levels of EC-SOD and adiponectin by the pioglitazone treatment indicated in Fig. 2 were consistent with the above observations. It has been reported that administration of pioglitazone normalized the overexpression of TNF- $\alpha$ and decreased the plasma TNF- $\alpha$ level in experimental animals (Hofmann et al. 1994, Murase et al. 1998). We also observed that the pioglitazone treatment significantly decreased the plasma TNF- $\alpha$ level in diabetic patients, as shown in Fig. 2C. The mechanism by which this occurs is unclear; however, TNF- $\alpha$ is known to suppress EC-SOD expression in cultured cells (Marklund 1992, Strålin \& Marklund 2000). From these results, it is speculated that the decline in the TNF- $\alpha$ level by the pioglitazone treatment results in an increase in EC-SOD expression in the vascular system.

In insulin-resistant patients, the decline in the activities of antioxidative enzymes (Cavarape et al. 2001) and the increase in superoxide production (Yamagishi et al. 2001) may lead to the progression of atherosclerosis via the enhancement of LDL oxidation (Heinecke et al. 1986). It has been reported that exogenous addition of EC-SOD and overexpression of EC-SOD prevented endothelial cell-mediated oxidative modification of LDL (Laukkanen et al. 2000, Takatsu et al. 2001). Moreover, mice lacking
EC-SOD were reported to be more prone to develop diabetes than wild-type controls (Sentman et al. 1999). The plasma TNF- $\alpha$ level was reported to be higher in patients with insulin resistance (Nilsson et al. 1998, Mishima et al. 2001) and correlated well with the degree of atherosclerosis, as shown by factors such as LDLcholesterol concentration (Skoog et al. 2002). In view of all these findings, it appears that both a decrease in EC-SOD expression and a decrease in the capability of the endothelial cell surface to possess EC-SOD in the diabetes/ insulin-resistant state probably lead to a decrease in the capability of EC-SOD to protect the endothelial cell function, and may increase atherosclerotic development in insulin-resistant patients. Increasing the EC-SOD expression might be one approach to ameliorate the pathogenesis of atherosclerosis in these patients.

\section{Acknowledgements}

This work was supported in part by a Grant-in-Aid for Scientific Research from the Japan Society for the Promotion of Science (to T A) and a grant for specific research from Gifu Pharmaceutical University (to T A)

\section{References}

Adachi T \& Marklund SL 1989 Interactions between human extracellular superoxide dismutase $\mathrm{C}$ and sulfated polysaccharides. Journal of Biological Chemistry 264 8537-8541.

Adachi T, Nakamura M, Yamada H, Futenma A, Kato K \& Hirano K 1994 Quantitative and qualitative changes of extracellular-superoxide dismutase in patients with various diseases. Clinica Chimica Acta 229 123-131.

Adachi T, Yamada H, Yamada Y, Morihara N, Yamazaki N, Murakami T, Futenma A, Kato K \& Hirano K 1996 Substitution of glycine for arginine-213 in extracellular-superoxide dismutase impairs affinity for heparin and endothelial cell surface. Biochemical Journal 313 235-239.

Bertelsen M, Änggård EE \& Carrier MJ 2001 Oxidative stress impairs insulin internalization in endothelial cells in vitro. Diabetologia 44 605-613.

Cavarape A, Feletto F, Mercuri F, Quagliaro L, Daman G \& Ceriello A 2001 High-fructose diet decreases catalase mRNA levels in rat tissues. Journal of Endocrinological Investigation 24 838-845.

Craven PA, Melhem MF, Phillips SL \& DeRubertis FR 2001 Overexpression of $\mathrm{Cu}^{2+} / \mathrm{Zn}^{2+}$ superoxide dismutase protects against early diabetic glomerular injury in transgenic mice. Diabetes $\mathbf{5 0}$ 2114-2125.

Faure P, Rossini E, Wiernsperger N, Richard MJ, Favier A \& Halimi S 1999 An insulin sensitizer improves the free radical defense system potential and insulin sensitivity in high fructose-fed rats. Diabetes 48 353-357.

Heinecke JW, Baker L, Rosen H \& Chait A 1986 Superoxide-mediated modification of low density lipoprotein by arterial smooth muscle cells. Journal of Clinical Investigation $\mathbf{7 7}$ 757-761.

Hofmann C, Lorenz K, Braithwaite SS, Colca JR, Palazuk BJ, Hotamisligil GS \& Spiegelman BM 1994 Altered gene expression for tumor necrosis factor- $\alpha$ and its receptors during drug and dietary modulation of insulin resistance. Endocrinology 134 264-270. 
Hotta K, Funahashi T, Arita Y, Takahashi M, Matsuda M, Okamoto Y, Iwahashi H, Kuriyama H, Ouchi N, Maeda K et al. 2000 Plasma concentrations of a novel, adipose-specific protein, adiponectin, in type 2 diabetic patients. Arteriosclerosis, Thrombosis, and Vascular Biology 20 1595-1599.

Iwata M, Haruta T, Usui I, Tanaka Y, Takano A, Uno T, Kawahara J, Ueno E, Sasaoka T, Ishibashi O \& Kobayashi M 2001 Pioglitazone ameliorates tumor necrosis factor- $\alpha$-induced insulin resistance by a mechanism independent of adipogenic activity of peroxisome proliferator-activated receptor- $\gamma$. Diabetes $\mathbf{5 0}$ 1083-1092.

Jain SK 1989 Hyperglycemia can cause membrane lipid peroxidation and osmotic fragility in human red blood cells. Journal of Biological Chemistry $26421340-21345$.

Karlsson K \& Marklund SL 1988 Extracellular superoxide dismutase in the vascular system of mammals. Biochemical Journal 255 223-228.

Koppenol WH, Moreno JJ, Pryor WA, Ischiropoulos H \& Beckman JS 1992 Peroxynitrite, a cloaked oxidant formed by nitric oxide and superoxide. Chemical Research in Toxicology 5 834-842.

Laukkanen MO, Lehtolainen P, Turunen P, Aittomäki S, Oikari P, Marklund SL \& Ylä-Herttuala S 2000 Rabbit extracellular superoxide dismutase: expression and effect on LDL oxidation. Gene 254 173-179.

Marklund SL 1990 Expression of extracellular superoxide dismutase by human cell lines. Biochemical Journal 266 213-219.

Marklund SL 1992 Regulation by cytokines of extracellular superoxide dismutase and other superoxide dismutase isoenzymes in fibroblasts. Journal of Biological Chemistry 267 6696-6701.

Marklund SL, Nilsson P, Israelsson K, Schampi I, Peltonen M \& Asplund K 1997 Two variants of extracellular-superoxide dismutase: relationship to cardiovascular risk factors in an unselected middle-aged population. Journal of Internal Medicine 242 5-14.

Matsuzawa Y, Funahashi T \& Nakamura T 1999 Molecular mechanism of metabolic syndrome $\mathrm{X}$ : contribution of adipocytokines adipocyte-derived bioactive substances. Annals of the New York Academy of Sciences 892 146-154.

Mishima Y, Kuyama A, Tada A, Takahashi K, Ishioka T \& Kibata M 2001 Relationship between serum tumor necrosis factor-alpha and insulin resistance in obese men with type 2 diabetes mellitus. Diabetes Research and Clinical Practice 52 119-123.

Murase K, Odaka H, Suzuki M, Tayuki N \& Ikeda H 1998 Pioglitazone time-dependently reduces tumor necrosis factor- $\alpha$ level in muscle and improves metabolic abnormalities in Wistar fatty rats. Diabetologia 41 257-264.

Nilsson J, Jovinge S, Niemann A, Reneland R \& Lithell H 1998 Relation between plasma tumor necrosis factor- $\alpha$ and insulin sensitivity in elderly men with non-insulin-dependent diabetes mellitus. Arteriosclerosis, Thrombosis, and Vascular Biology 18 $1199-1202$
Nishikawa T, Edelstein D, Du XL, Yamagishi S, Matsumura T, Kaneda Y, Yorek MA, Beebe D, Oates PJ, Hammes H-P et al. 2000 Normalizing mitochondrial superoxide production blocks three pathways of hyperglycaemic damage. Nature 404 787-790.

Nishizawa H, Shimomura I, Kishida K, Maeda N, Kuriyama H, Nagaretani H, Matsuda M, Kondo H, Furuyama N, Kihara S et al. 2002 Androgens decrease plasma adiponectin, an insulin-sensitizing adipocyte-derived protein. Diabetes 51 2734-2741.

Ohara Y, Peterson TE \& Harrison DG 1993 Hypercholesterolemia increases endothelial superoxide anion production. Journal of Clinical Investigation 91 2546-2551.

Pieper GM, Meier DA \& Hager SR 1995 Endothelial dysfunction in a model of hyperglycemia and hyperinsulinemia. American Journal of Physiology 269 H845-H850.

Sentman M-L, Jonsson LM \& Marklund SL 1999 Enhanced alloxan-induced $\beta$-cell damage and delayed recovery from hyperglycemia in mice lacking extracellular-superoxide dismutase. Free Radical Biology and Medicine 27 790-796.

Skoog T, Dichtl W, Boquist S, Skoglund-Andersson C, Krape F, Tang R, Bond MG, de Faire U, Nilsson J, Eriksson P \& Hamsten A 2002 Plasma tumour necrosis factor-alpha and early carotid atherosclerosis in healthy middle-aged men. European Heart Journal 23 376-383.

Strålin P \& Marklund SL 2000 Multiple cytokines regulate the expression of extracellular superoxide dismutase in human vascular smooth muscle cells. Atherosclerosis 151 433-441.

Strålin P, Karlsson K, Johansson BO \& Marklund SL 1995 The interstitium of the human arterial wall contains very large amounts of extracellular superoxide dismutase. Arteriosclerosis, Thrombosis, and Vascular Biology 15 2032-2036.

Takatsu H, Tasaki H, Kim HN, Ueda S, Tsutsui M, Yamashita K, Toyokawa T, Morimoto Y, Nakashima Y \& Adachi T 2001 Overexpression of EC-SOD suppresses endothelial-cell-mediated LDL oxidation. Biochemical and Biophysical Research Communications 285 84-91.

Wink DA, Hanbauer I, Krishna MC, DeGraff W, Gamson J \& Mitchell JB 1993 Nitric oxide protects against cellular damage and cytotoxicity from reactive oxygen species. PNAS 90 9813-9817.

Yamagishi S, Edelstein D, Du X-L, Kaneda Y, Guzmán M \& Brownlee M 2001 Leptin induces mitochondrial superoxide production and monocyte chemoattractant protein-1 expression in aortic endothelial cells by increasing fatty acid oxidation via protein kinase A. Journal of Biological Chemistry 276 25096-25100.

Received 26 December 2003

Accepted 11 February 2004 